\title{
DNA damage response and evasion from immunosurveillance in CLL: new options for NK cell-based immunotherapies
}

\author{
Olga M. Shatnyeva, Hinrich P. Hansen, Katrin S. Reiners, Maike Sauer, Maulik Vyas and \\ Elke Pogge von Strandmann*
}

Innate Immunity Group, Clinic 1 for Internal Medicine, University of Cologne, Cologne, Germany

Edited by:

Christian Reinhardt, Uniklinik Köln,

Germany

Reviewed by:

Jian-Jun Wei, Northwestern

University, USA

Massimo Broggini, Istituto di

Ricerche Farmacologiche Mario

Negri, Italy

\section{*Correspondence:}

Elke Pogge von Strandmann, Innate

Immunity Group, Clinic 1 for

Internal Medicine, University of

Cologne, Kerpener Straße 62,

50924 Cologne, Germany

e-mail: elke.pogge@uk-koeln.de
Chronic lymphocytic leukemia $(C L L)$ is the most prominent $B$ cell malignancy among adults in the Western world and characterized by a clonal expansion of B cells. The patients suffer from severe immune defects resulting in increased susceptibility to infections and failure to generate an antitumor immune response. Defects in both, DNA damage response (DDR) pathway and crosstalk with the tissue microenvironment have been reported to play a crucial role for the survival of CLL cells, therapy resistance and impaired immune response. To this end, major advances over the past years have highlighted several $\mathrm{T}$ cell immune evasion mechanisms in CLL. Here, we discuss the consequences of an impaired DDR pathway for detection and elimination of CLL cells by natural killer (NK) cells. NK cells are considered to be a major component of the immunosurveillance in leukemia but NK cell activity is impaired in CLL. Restoration of NK cell activity using immunoligands and immunoconstructs in combination with the conventional chemotherapy may provide a future perspective for CLL treatment.

Keywords: chronic lymphocytic leukemia, DNA damage response, natural killer cell, immunotherapy immunoligands, immunoconstructs

\section{INTRODUCTION}

Chronic lymphocytic leukemia (CLL) is an indolent lymphoproliferative disorder characterized by the progressive accumulation of monoclonal $\mathrm{CD}^{+} \mathrm{B}$ cells in the peripheral blood, bone marrow and secondary lymphoid tissues (Ghia and Hallek, 2014; Yair et al., 2014). Another typical feature of CLL is extraordinary high frequency of chromosomal aberrations often associated with the DNA damage response (DDR) pathway and dysregulation of the cell cycle (Landau et al., 2013). Recent data suggest that defects of the DDR pathway and interaction with bystander cells of the microenvironment are pivotal factors for CLL progression. Prolonged DNA damage and defective repair results in the release of DNA-HMGB1 complexes from necrotic cells and the induction of inflammatory response that can be hijacked by CLL cells (Jia et al., 2014).

The standard therapy of CLL which includes combined chemotherapy and/or immunotherapy is highly efficient for the depletion of CLL cells from the peripheral blood but not from lymphoid tissue and bone marrow. Moreover, this might result in the selection of resistant clones (Landau et al., 2013). Haploidentical stem cell transplantation (HSCT) is an alternative treatment but restricted to a limited group of patients due to lack of suitable donors and may cause fatal side effects. New successful therapeutic strategies include modulation of the microenvironment and activation of the patient immune system to combat cancer cells (Burger and Gribben, 2014; Yair et al., 2014).
Direct targeting of tumor-associated antigens (TAA) on malignant cells by monoclonal antibodies $(\mathrm{mAb})$ is regarded as a promising approach (Simpson and Caballero, 2014). Thus, antibodies against CD20 (rituximab, Obinutuzumab), CD19 (GBR 401), CD23 (lumiliximab), or CD52 (alemtuzumab) are currently evaluated (Robak, 2013). More recently, immunotherapies with genetically engineered chimeric T-cell receptors (CARs), which detect TAA, were developed (Burger and Gribben, 2014; Yair et al., 2014). So far, $T$ cells with specificity for the common B cell antigens CD19, CD20, and CD23 were generated (Riches and Gribben, 2013). Initial clinical trials revealed feasibility, and increasingly also impressive antitumor effects.

There is emerging evidence that natural killer (NK) cells also play a pivotal role in the immunosurveillance of CLL (Reiners et al., 2013; Huergo-Zapico et al., 2014). Understanding of the molecular mechanisms of evasion from NK cell-mediated immune responses and recovery of their function will help to develop novel treatment strategies. In this review we focus on the role of DDR defects and the immune microenvironment in the evasion from NK cell responses in CLL, as far as on restoration of NK cell function using immunoligands and immunoconstructs.

\section{THE ROLE OF DDR DEFECTS IN CLL DEVELOPMENT: IMPACT ON ESCAPE FROM NK CELL IMMUNE RESPONSE}

Recent studies have identified 20 candidate CLL driver genes associated with core signaling pathways, including DNA repair, cell cycle control, Notch signaling, inflammatory pathways, Wnt 
signaling, RNA splicing, and RNA processing (Landau et al., 2013), among which mutations associated with DNA repair and cell cycle regulation are most recurrent.

The physiological function of the DDR pathway is to detect DNA damage, to signal its presence and to mediate DNA repair. The proximal DDR constitutes of two major kinase branches, the ATM/Chk2 and the ATR/Chk1 pathways. Activation of ATR, which phosphorylates its effector kinase Chk1, is induced in response to single-strand breaks and bulky DNA lesions. The ATM kinase, signaling through its effector Chk2, is activated primarily in response to DNA double-strand breaks (DSBs), such as those induced by alkylating agents, topoisomerase inhibitors, or ionizing radiation. Chk1 and Chk2 have a protective function providing time to the cell to repair genotoxic lesions. Both kinase pathways result in activation of cell cycle-arresting target genes, DNA repair and apoptosis via p53 activation (Reinhardt and Yaffe, 2009).

Recent sequencing studies identified recurrent somatic gene mutations in CLL patients for proteins involved in DNA damage signaling and DNA repair, including mutations in TP53, ATM, CHEK1, CHEK2, POT1, BRCA1, and CHD2 (Puente et al., 2011; Quesada et al., 2011).

The ATM-Chk2-p53 signaling axis plays an important role in regulation of apoptotic response to DNA damage in CLL, as mutations in ATM and TP53 are enriched in patients with secondary resistance to DNA-damaging chemotherapy (Bartkova et al., 2005; Landau et al., 2013).

ATM gene is frequently inactivated in CLL and is associated with defective apoptosis in response to chemotherapeutic agents (Austen et al., 2007). ATM mutant cells exhibit impaired DNA DSB repair. Poly (ADP-ribose) polymerase (PARP) plays a pivotal role in a direct repair of DSBs and involved in main DNA repair mechanisms: homologous recombination and non-homologous end-joining (Weston et al., 2010). ATM dysfunction is associated with significantly higher PARP activity in CLL patients, which might mediate genomic instability and progression of the disease. In vivo studies using xenograft model of an ATM mutant cell line demonstrated significantly reduced tumor load and an increased survival of animals after treatment with the PARP inhibitor Olaparib (Weston et al., 2010). Clinical studies with Olaparib demonstrated sufficient efficacy in patients with ATM deficient, relapsed and refractory CLL (ISRCTN34386131 DOI 10.1186/ISRCTN34386131). Deletions of the short arm of chromosome $17(\operatorname{del}(17 \mathrm{p}))$ where TP53 is located are found in 5-8\% of chemotherapy-naïve patients (Dohner et al., 2000). Mutations of TP53 are found in $4-37 \%$ of patients with CLL, and have been associated with very poor prognosis (ultra-high risk) in a number of studies (Zenz et al., 2010). Among cases with confirmed $\operatorname{del}(17 \mathrm{p})$, the majority show mutations in the remaining TP53 allele (>80\%). Higher genomic complexity and clinical diversity of CLL are associated with T53 mutations. Impaired DDR promotes a "mutator phenotype," which allows the acquisition of additional genetic lesions driving transformation in CLL (Seiffert et al., 2012).

Mutational inactivation of the DDR is an established hallmark of CLL and associated with high genomic instability (Zenz et al., 2010; Landau et al., 2013).
ATM appears to be a major regulator of the p53 response. They communicate the genotoxic lesion to the apoptotic machinery but they are frequently inactivated in CLL and are associated with poor response to conventional chemotherapy (ten Hacken and Burger, 2014).

The $\mathrm{B}$ cell receptor (BCR) pathway inhibitors in CLL have shown high efficacy in the cases with poor chromosomal aberrations such as Del (17p) or p53 mutation, known to acquire resistance to standard chemotherapy. Downstream targets of the BCR such as SYK, Bruton's tyrosine kinase (BTK), or PI3K isoform p110 delta have a promising anti-neoplastic activity in patients with CLL. Responses are typically manifested by rapid regression of enlarged lymph nodes and splenomegaly that is accompanied by transient lymphocytosis (Burger and Gribben, 2014; Yair et al., 2014).

Clinical trials with Idelalisib, PI3K delta isoform inhibitor, have a dramatic and durable response in CLL patients with a markers of poor prognosis, such as mutations in p53, ATM and NOTCH1. Monotherapy with Idelalisib and combination with other therapeutical agents such as Rituximab and Ofatumumab results show good activity in CLL regardless of high-risk prognostic markers (Khan et al., 2014).

Moreover, the DDR is able to alert the immune system toward the stressed cell, mainly through the recruitment of NK cells, which are able to identify and eliminate dangerous cells without prior antigen-mediated stimulation (Raulet, 2006; Bryceson and Ljunggren, 2008). NK cells do not only distinguish between "self" and "non-self," but specifically seek for pathological changes in endogenous cells. One important danger signal is the inducible expression of ligands for cytotoxic NK cell receptors [NKG2D (NK group 2, member D) and NCRs (natural cytotoxicity receptors)] to alarm the innate immune system in response to DNA damage (Gasser et al., 2005; Gasser and Raulet, 2006a,b,c; Gasser, 2007; Soriani et al., 2009; Fine et al., 2010; Norman et al., 2011). Ligands for these NK cell receptors are not expressed on normal cells but are found on cells undergoing cellular stress that causes DNA damage including chemotherapeutics or ionizing radiation (Raulet, 2006).

The expression of NKG2D ligands in response to genotoxic stress and stalled DNA replication forks is induced through canonical DDR in an ATM/ATR-dependent fashion in mouse and human fibroblasts (Gasser et al., 2005). The NKp30 ligand BAG6 is released by stressed cells via the exosomal pathway and has to be associated with these small membrane vesicles to properly activate NK cells (Simhadri et al., 2008). The release of exosomes is known to be regulated by TSAP6 in a p53-dependent manner (Lespagnol et al., 2008). Thus, defects in the DDR such as p53 mutations may directly affect NK cell-dependent recognition and elimination of CLL cells (Reiners et al., 2013). In line, an impaired expression of ligands for two major activating receptors-NKG2D and NKp30 - was shown to be associated with CLL probably explaining NK cell anergy in this disease (Figure 1; Salih et al., 2008; Nuckel et al., 2010; Costello et al., 2012; Reiners et al., 2013). However, mechanisms of escape from NK response in CLL are not completely clear, but defects in NK cell activity strongly correlate with progression of the disease (Ziegler et al., 1981; Riches and Gribben, 2013). 

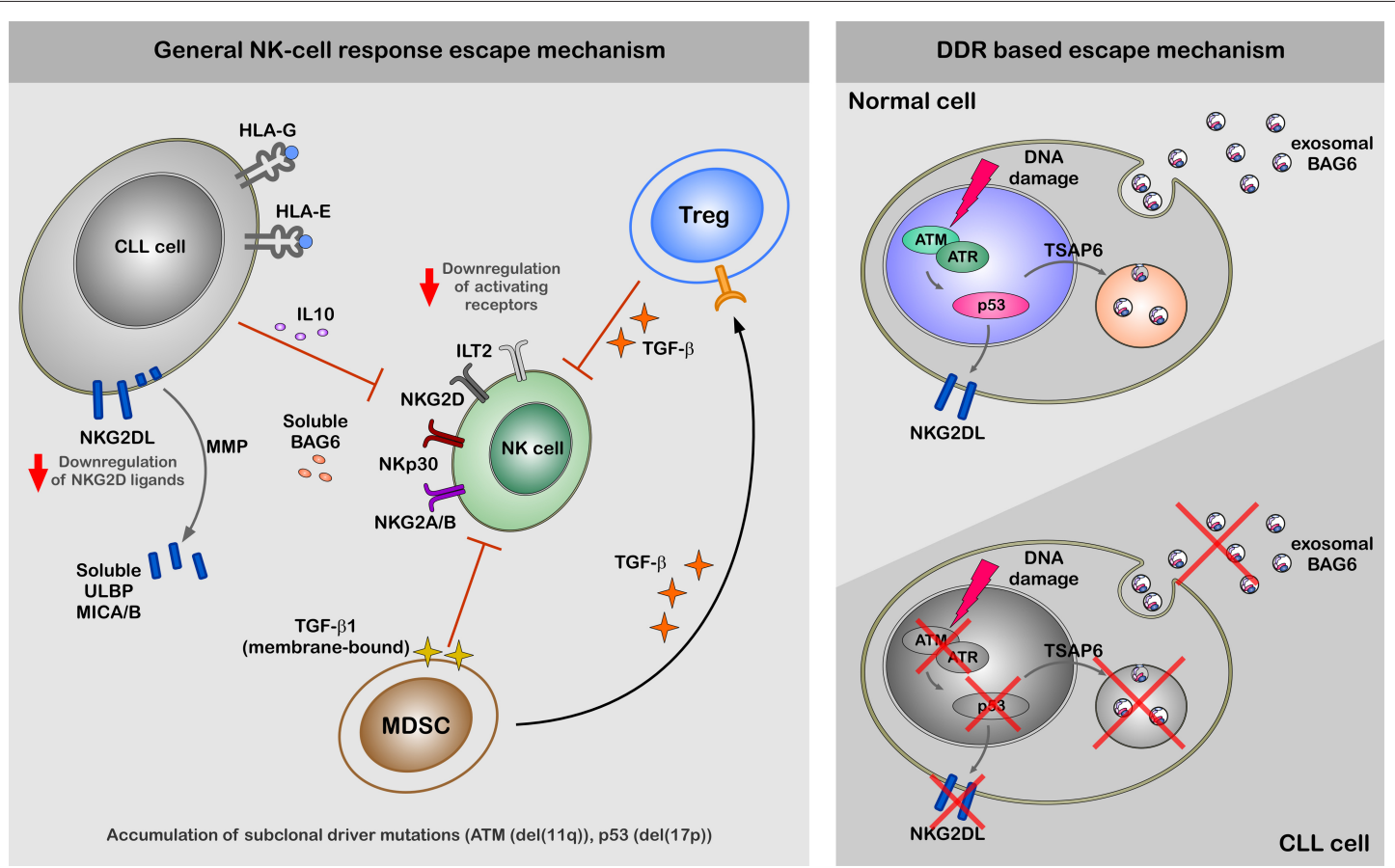

FIGURE 1 | A hypothetical model of CLL escape from NK response. The left panel of the figure demonstrate general mechanism of the escape from NK-cell response by CLL cells. The escape of CLL cells from the NK cell response is regulated at different levels; (1) diminished expression NKG2D ligands on the cell surface, (2) increased levels of ligands for inhibitory receptors ILT2 and NKG2A/B, (3) shedding by MMPs and production of soluble ligands for activating receptors (ULBP2, MICA, and BAG6) NKG2D and NKp30 (Iclozan et al., 2013), which rather suppress than activate NK cells. Indirect suppression of NK cell activity might be regulated by MDSC and Treg cells via production of TGF $\beta$ and IL-10, which modulate expression levels of activating receptors on the cell surface of NK cells. The right panel of the figure is focused on the impaired DDR and its role for the escape from NK-cell response. Induction of DDR in healthy cells results in activation ATM-p53 axis. Activation of p53 results in cell surface expression of NKG2D ligands and exosomal release of BAG6 following transcriptional activation of TSAP6. Cell surface expression of NKG2D ligands and exosomal expression of BAG6 is impaired in CLL cells due to defects in DDR.

\section{IMMUNE MICROENVIRONMENT DRIVES CLL PROGRESSION}

The tumor microenvironment plays an important role in CLL progression. The functional components of the microenvironment can be divided in three groups. The first group includes bone marrow stromal cells (BMSC), nurse-like cells (NLC), and follicular dendritic cells (FDCs), collectively involved in supporting selection, survival and proliferation of CLL cells. BMSCs send anti-apoptotic signals via VCAM and integrins and protect CLL cells against conventional chemotherapy (Gehrke et al., 2011). NLC attract CLL cells by secreting CXCL12 and CXCL13 and protect from drug induced apoptosis via CXCL12, BAFF, APRIL, $\mathrm{CD} 31$, and plexin-B via activation of prosurvival cascades such as NFkB and ERK (Burger and Gribben, 2014). Also NLCs play important role in activation of the BCR signaling cascade. FDCs protect CLL cells from apoptosis by direct contact resulting in upregulation of antiapoptotic protein MCL-1 (Endo et al., 2007).

The second group is represented by regulatory $\mathrm{T}$ cells (Treg) and myeloid-derived suppressor cells (MDSCs). They interfere with the complex interaction between the immune system and transformed cells via production of immunosuppressive soluble factors such as TGF- $\beta$ and IL-10 (D'Arena et al., 2013; Jitschin et al., 2014).

The third group encompasses components of the immune system, including $\mathrm{CD} 4^{+} \mathrm{T}, \mathrm{CD} 8^{+} \mathrm{T}$, and NK cells. Despite an elevated $\mathrm{T}$ cell count in the peripheral blood, the $\mathrm{T}$ cell compartment is abnormal in CLL, showing profound functional defects and signs of chronic activation [upregulation of CD69, HLA-DR, and CD57 and downregulation of CD28 and CD62L (Pedersen et al., 2002; Burger and Gribben, 2014)]. CD4 ${ }^{+}$T cells stimulate CLL cells via CD40/CD40L crosstalk to induce the production of CCL17 and CCL22 for attracting Th2 lymphocytes (Nakayama et al., 2004). The NK cell subset in CLL patients shows reduced ability to attack cancer cells partly owing to diminished expression of the activating NK receptor NKp30 on the cell surface (Costello et al., 2012). Also, HLA-G and HLA-E on the CLL cells and high levels of soluble/decoy ligands, for activating NK cell receptors suppress NK cell function (Nuckel et al., 2005).

\section{NOVEL APPROACHES FOR CLL TREATMENT: IMMUNE CONSTRUCTS AS TOOLS TO REDIRECT NK CELLS AGAINST TUMOR CELLS}

The improved understanding of the pathology of CLL and the role of the microenvironment resulted in the development of novel less toxic agents (Burger and Gribben, 2014; Yair et al., 2014). These new compounds include inhibitors aimed at BCR signaling pathway (Pallasch and Hallek, 2014), antiapoptotic proteins (Yair et al., 2014), mAbs (Robak, 2013), and immune-modulatory drugs (Burger and Gribben, 2014; Yair et al., 2014). 


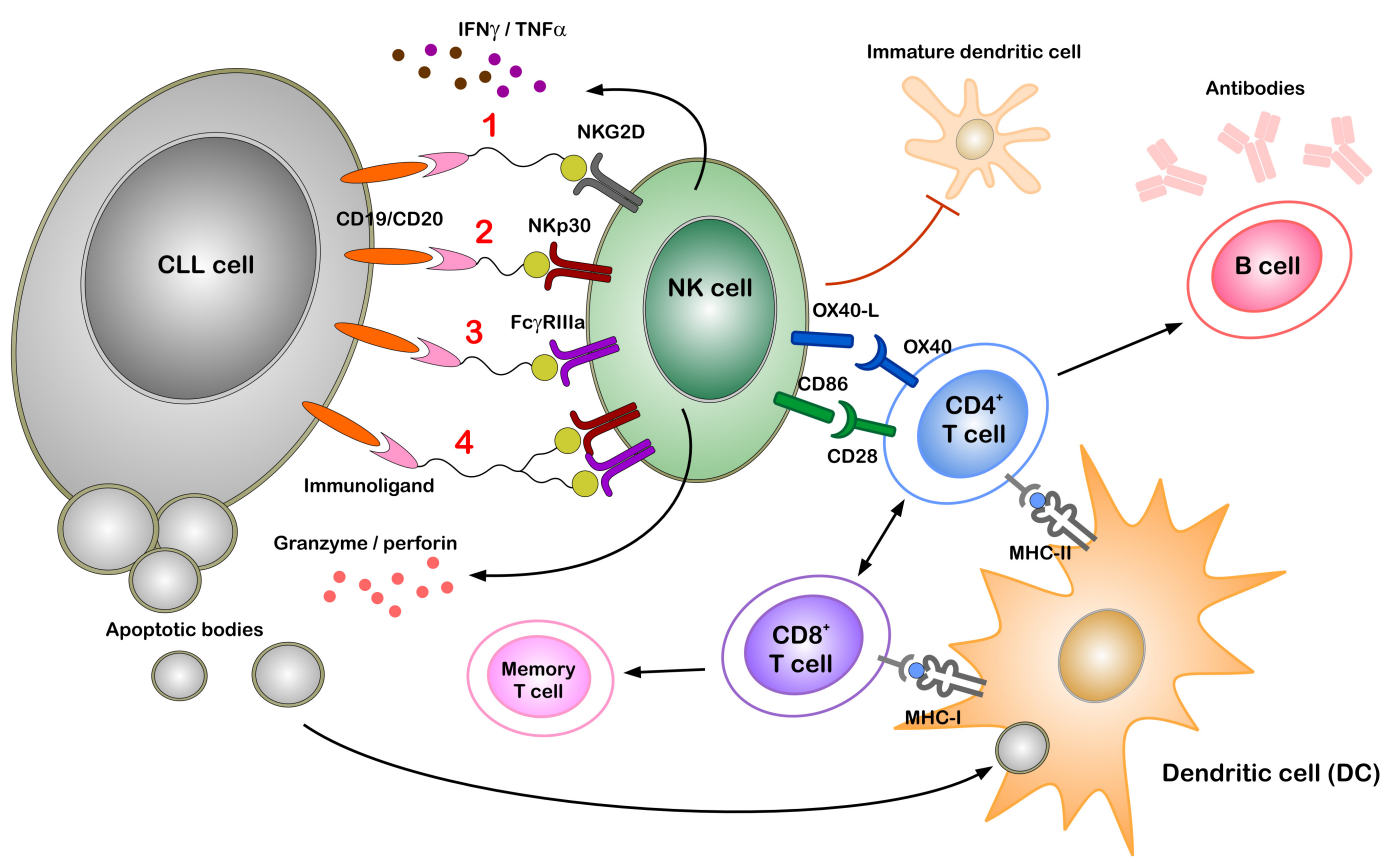

FIGURE 2 | Potential role of immunoligands to redirect NK cells to CLL cells. Immunoligands with specificities for CLL cells (through CD19 or CD20) and for NK cells [through activating receptors on NK cells such as NKG2D, NKp30, Fc $\gamma$ RIIla or simultaneous NKp30 and Fc $\gamma$ RIIIa(1-4)] can link and activate respective immune cells even in the presence of active immune suppression. Stimulating of either of the activating receptors on NK cell leads to cytokine secretion (IFN $\gamma, \mathrm{TNF} \alpha$ ) and degranulation, thereby killing tumor cells by apoptosis. Professional antigen presenting cells (APCs) such as dendritic cells (DCs) phagocytose components of dying tumor cells and present tumor antigens to both $\mathrm{CD}^{+}$and $\mathrm{CD} 4^{+} \mathrm{T}$ cells, thereby inducing cell-mediated and humoral adaptive immunity and memory response. Direct NK-DC crosstalk in terms of maturation of DCs and killing of immature DCs is mainly attributed to NKG2D and NKp30 activation. Additionally, following activation NK cells express OX40 ligand (OX40L) and CD86 on the cell surface, which can bind to the co-stimulatory receptors OX40 and CD28 expressed by CD4 ${ }^{+}$T cells. Direct interaction between NK and $\mathrm{CD} 4^{+} \mathrm{T}$ cells through such co-stimulatory molecules can enhance $\mathrm{T}$ cell effector functions.
Current immunotherapy for CLL is mainly intended to stimulate $\mathrm{T}$ cells in order to eliminate the tumor (Costello et al., 2012; Burger and Gribben, 2014), whereas NK cell-based therapies are not as advanced. Various recombinant immunoligands and immune constructs to restore impaired NK cell activity have been developed and analyzed pre-clinically. While both represent recombinant constructs, the immunoligands utilize natural ligands for immune receptors fused to tumor-specific single chain variable fragment $(\mathrm{scFv})$ or other antibody-derived fragments whereas the immune constructs utilize antibody-derived components to target both immune and target cells (Vyas et al., 2014). So far, the activating receptors Fc $\gamma$ RIIIa, NKG2D, and NKp30 were used as target structures on NK cells.

FcyRIIIa (CD16a) s is one of the main NK cell-activating receptors, which upon stimulation, mediates ADCC through the release of granzyme and perforin (Alderson and Sondel, 2011). The clinical success of many FDA-approved mAbs (e.g., Rituximab) is partially attributed to NK cell-mediated ADCC through Fc $\gamma$ RIIIa receptor (Houot et al., 2011). Several bispecific and trispecific immunoconstructs with one arm specific for the human Fc $\gamma$ RIIIa receptor have been developed. These constructs target different tumor antigens (CD33, CD123, and CD19) and are currently tested for effectiveness (Singer et al., 2010; Stein et al., 2010).
NKG2D is known as the most important receptor involved in immune evasion of CLL and other tumor cells (HuergoZapico et al., 2014). Tumor cells downregulate the expression of the ligands for NKG2D and release soluble ligands upon induced shedding by matrix metalloproteinases (MMPs) that block NKG2D receptor function (Huang et al., 2011; Chitadze et al., 2013). Immunoligands containing human NKG2D ligands have already been generated (Germain et al., 2005; Pogge von Strandmann et al., 2006; Jachimowicz et al., 2011; Kellner et al., 2012b; Rothe et al., 2014). One of them is a recombinant bispecific immunoligand bearing ULBP2 ligand-scFv fusion in a single chain format. The first construct of this kind was ULBP2-BB4, which links NK cells and CD138 ${ }^{+}$tumor cells through the ULBP2 ligand and the BB4 scFv with specificity for the TAA CD138 (Pogge von Strandmann et al., 2006). ULBP2-BB4 successfully activated and retargeted NK cells against multiple myeloma (MM) tumor cell lines and primary patient tumor cells and showed antitumor activity in a xenograft MM model. Another bispecific protein developed consists of recombinant MICA as NKG2D ligand chemically conjugated to Fab fragments from mAb specific for TAAs such as CD19 and CD20 (Kellner et al., 2012b). Malignant cells, which are otherwise resistant, can be rendered susceptible to NK cell attack by NKG2D ligand MICA in this format (Germain et al., 2005). 
Targeting NKp30 to reactivate NK cells in CLL is most promising and feasible as novel activating ligands for NKp30 are identified recently. While BAG6 can activate NK cells via NKp30 to induce target killing of tumor cells and immature DCs when expressed on the surface of exosomes (Pogge von Strandmann et al., 2007; Simhadri et al., 2008), expression of B7-H6 seems to be more tumor-confined as it is also found on the surface of cancer cells (Brandt et al., 2009; Matta et al., 2013). A bispecific immunoligand (B7-H6-7D8) comprising the B7-H6 (NKp30 ligand) ectodomain fused to a 7D8-derived anti-CD20 scFv was generated (Kellner et al., 2012a). The B7-H6-7D8 immunoligand efficiently redirected NKp30-dependent NK cell mediated lysis toward CD20 ${ }^{+}$lymphoma cells (Kellner et al., 2012a, 2013). Furthermore, this construct enhanced the lysis of target cells when used along with either rituximab (anti-CD20 mAb which activates the FcgRIIIa receptor) or NKG2D activating construct ULBP27D8 (Kellner et al., 2012a, 2013). The concept of synergistic activation of NK cells by multiple activating receptors is proven in various systems in vitro (Bryceson et al., 2006; Morgado et al., 2011; Deguine et al., 2012) and will be applied in the next generation of immunoligands in a triplebody format (Vyas et al., 2014; Figure 2).

\section{CONCLUDING REMARKS AND FUTURE PERSPECTIVES}

In the last decade a better understanding of the role of the immune environment in progression of CLL led to new targeted therapy options. NK cells are the first line to attack the cancer, but their activity is highly impaired in CLL patients. Recent studies show impressive pre-clinical responses to immunoligands and immunoconstructs redirecting NK cells against different cancers. Novel immune construct-mediated restoration of NK cell functionality in CLL patients is an attractive strategy for treatment, which might even pave the way for recovery of patients with high risk cytogenetic aberrations and/or refractory disease.

\section{ACKNOWLEDGMENTS}

This study was supported by the Deutsche Forschungsgemeinschaft (KFO286) (grant to Elke Pogge von Strandmann), by a grant of the Deutsche Krebshilfe to Elke Pogge von Strandmann and Köln Fortune to Olga M. Shatnyeva. We thank Andriy Kubarenko and Pranjali Bhagwat for critical reading of the manuscript.

\section{REFERENCES}

Alderson, K. L., and Sondel, P. M. (2011). Clinical cancer therapy by NK cells via antibody-dependent cell-mediated cytotoxicity. J. Biomed. Biotechnol. 2011, 379123. doi: $10.1155 / 2011 / 379123$

Austen, B., Skowronska, A., Baker, C., Powell, J. E., Gardiner, A., Oscier, D., et al. (2007). Mutation status of the residual ATM allele is an important determinant of the cellular response to chemotherapy and survival in patients with chronic lymphocytic leukemia containing an 11q deletion. J. Clin. Oncol. 25, 5448-5457. doi: 10.1200/JCO.2007.11.2649

Bartkova, J., Horejsi, Z., Koed, K., Kramer, A., Tort, F., Zieger, K., et al. (2005). DNA damage response as a candidate anti-cancer barrier in early human tumorigenesis. Nature 434, 864-870. doi: 10.1038/nature03482

Brandt, C. S., Baratin, M., Yi, E. C., Kennedy, J., Gao, Z., Fox, B., et al. (2009). The B7 family member B7-H6 is a tumor cell ligand for the activating natural killer cell receptor NKp30 in humans. J. Exp. Med. 206, 1495-1503. doi: 10.1084/ jem. 20090681
Bryceson, Y. T., and Ljunggren, H. G. (2008). Tumor cell recognition by the NK cell activating receptor NKG2D. Eur. J. Immunol. 38, 2957-2961. doi: 10.1002/ eji.200838833

Bryceson, Y. T., March, M. E., Ljunggren, H. G., and Long, E. O. (2006). Synergy among receptors on resting NK cells for the activation of natural cytotoxicity and cytokine secretion. Blood 107, 159-166. doi: 10.1182/blood-2005-04-1351

Burger, J. A., and Gribben, J. G. (2014). The microenvironment in chronic lymphocytic leukemia (CLL) and other B cell malignancies: insight into disease biology and new targeted therapies. Semin. Cancer Biol. 24, 71-81. doi: 10.1016/ j.semcancer.2013.08.011

Chitadze, G., Lettau, M., Bhat, J., Wesch, D., Steinle, A., Furst, D., et al. (2013). Shedding of endogenous MHC class I-related chain molecules A and B from different human tumor entities: heterogeneous involvement of the "a disintegrin and metalloproteases” 10 and 17. Int. J. Cancer 133, 1557-1566. doi: 10.1002/ ijc. 28174

Costello, R. T., Knoblauch, B., Sanchez, C., Mercier, D., Le Treut, T., and Sebahoun, G. (2012). Expression of natural killer cell activating receptors in patients with chronic lymphocytic leukaemia. Immunology 135, 151-157. doi: 10.1111/j.13652567.2011.03521.x

D’Arena, G., Simeon, V., D’Auria, F., Statuto, T., Sanzo, P. D., Martino, L. D., et al. (2013). Regulatory T-cells in chronic lymphocytic leukemia: actor or innocent bystander? Am. J. Blood Res. 3, 52-57.

Deguine, J., Breart, B., Lemaitre, F., and Bousso, P. (2012). Cutting edge: tumortargeting antibodies enhance NKG2D-mediated NK cell cytotoxicity by stabilizing NK cell-tumor cell interactions. J. Immunol. 189, 5493-5497. doi: 10.4049/ jimmunol.1202065

Dohner, H., Stilgenbauer, S., Benner, A., Leupolt, E., Krober, A., Bullinger, L., et al. (2000). Genomic aberrations and survival in chronic lymphocytic leukemia. N. Engl. J. Med. 343, 1910-1916. doi: 10.1056/NEJM200012283432602

Endo, T., Nishio, M., Enzler, T., Cottam, H. B., Fukuda, T., James, D. F., et al. (2007). BAFF and APRIL support chronic lymphocytic leukemia B-cell survival through activation of the canonical NF-кB pathway. Blood 109, 703-710. doi: 10.1182/blood-2006-06-027755

Fine, J. H., Chen, P., Mesci, A., Allan, D. S., Gasser, S., Raulet, D. H., et al. (2010). Chemotherapy-induced genotoxic stress promotes sensitivity to natural killer cell cytotoxicity by enabling missing-self recognition. Cancer Res. 70, 71027113. doi: 10.1158/0008-5472.CAN-10-1316

Gasser, S. (2007). DNA damage response and development of targeted cancer treatments. Ann. Med. 39, 457-464. doi: 10.1080/07853890701436773

Gasser, S., Orsulic, S., Brown, E. J., and Raulet, D. H. (2005). The DNA damage pathway regulates innate immune system ligands of the NKG2D receptor. Nature 436, 1186-1190. doi: 10.1038/nature03884

Gasser, S., and Raulet, D. H. (2006a). The DNA damage response, immunity and cancer. Semin. Cancer Biol. 16, 344-347. doi: 10.1016/j.semcancer.2006.07.004

Gasser, S., and Raulet, D. H. (2006b). Activation and self-tolerance of natural killer cells. Immunol. Rev. 214, 130-142. doi: 10.1111/j.1600-065X.2006.00460.x

Gasser, S., and Raulet, D. H. (2006c). The DNA damage response arouses the immune system. Cancer Res. 66, 3959-3962. doi: 10.1158/0008-5472.CAN-05-4603

Gehrke, I., Gandhirajan, R. K., Poll-Wolbeck, S. J., Hallek, M., and Kreuzer, K. A. (2011). Bone marrow stromal cell-derived vascular endothelial growth factor (VEGF) rather than chronic lymphocytic leukemia (CLL) cell-derived VEGF is essential for the apoptotic resistance of cultured CLL cells. Mol. Med. 17, 619627. doi: 10.2119/molmed.2010.00210

Germain, C., Larbouret, C., Cesson, V., Donda, A., Held, W., Mach, J. P., et al. (2005). MHC class I-related chain A conjugated to antitumor antibodies can sensitize tumor cells to specific lysis by natural killer cells. Clin. Cancer Res. 11, 7516-7522. doi: 10.1158/1078-0432.CCR-05-0872

Ghia, P., and Hallek, M. (2014). Management of chronic lymphocytic leukemia. Haematologica 99, 965-972. doi: 10.3324/haematol.2013.096107

Houot, R., Kohrt, H. E., Marabelle, A., and Levy, R. (2011). Targeting immune effector cells to promote antibody-induced cytotoxicity in cancer immunotherapy. Trends Immunol. 32, 510-516. doi: 10.1016/j.it.2011.07.003

Huang, B., Sikorski, R., Sampath, P., and Thorne, S. H. (2011). Modulation of NKG2D-ligand cell surface expression enhances immune cell therapy of cancer. J. Immunother. 34, 289-296. doi: 10.1097/CJI.0b013e31820elb0d

Huergo-Zapico, L., Acebes-Huerta, A., Gonzalez-Rodriguez, A. P., Contesti, J., Gonzalez-Garcia, E., Payer, A. R., et al. (2014). Expansion of NK cells and reduction of NKG2D expression in chronic lymphocytic leukemia. Correlation with progressive disease. PLoS ONE 9:e108326. doi: 10.1371/journal.pone.0108326 
Iclozan, C., Antonia, S., Chiappori, A., Chen, D. T., and Gabrilovich, D. (2013). Therapeutic regulation of myeloid-derived suppressor cells and immune response to cancer vaccine in patients with extensive stage small cell lung cancer. Cancer Immunol. Immunother. 62, 909-918. doi: 10.1007/s00262-013-1396-8

Jachimowicz, R. D., Fracasso, G., Yazaki, P. J., Power, B. E., Borchmann, P., Engert, A., et al. (2011). Induction of in vitro and in vivo NK cell cytotoxicity using high-avidity immunoligands targeting prostate-specific membrane antigen in prostate carcinoma. Mol. Cancer Ther. 10, 1036-1045. doi: 10.1158/15357163.MCT-10-1093

Jia, L., Clear, A., Liu, F. T., Matthews, J., Uddin, N., McCarthy, A., et al. (2014), Extracellular HMGB1 promotes differentiation of nurse-like cells in chronic lymphocytic leukemia. Blood 123, 1709-1719. doi: 10.1182/blood-2013-10529610

Jitschin, R., Braun, M., Buttner, M., Dettmer-Wilde, K., Bricks, J., Berger, J., et al. (2014). CLL-cells induce IDO ${ }^{\text {hi }}$ CD14 ${ }^{+}$HLA-DR $^{\text {lo }}$ myeloid-derived suppressor cells that inhibit T-cell responses and promote TRegs. Blood 124, 750-760. doi: 10.1182/blood-2013-12-546416

Kellner, C., Gramatzki, M., and Peipp, M. (2013). Promoting natural killer cell functions by recombinant immunoligands mimicking an induced self phenotype. Oncoimmunology 2, e24481. doi: 10.4161/onci.24481

Kellner, C., Maurer, T., Hallack, D., Repp, R., van de Winkel, J. G., Parren, P. W., et al. (2012a). Mimicking an induced self phenotype by coating lymphomas with the NKp30 ligand B7-H6 promotes NK cell cytotoxicity. J. Immunol. 189, 50375046. doi: 10.4049/jimmunol.1201321

Kellner, C., Zhukovsky, E. A., Potzke, A., Bruggemann, M., Schrauder, A., Schrappe, M., et al. (2012b). The Fc-engineered CD19 antibody MOR208 (XmAb5574) induces natural killer cell-mediated lysis of acute lymphoblastic leukemia cells from pediatric and adult patients. Leukemia 27, 1595-1598. doi: 10.1038/leu.2012.373

Khan, M., Saif, A., Sandler, S., and Mirrakhimov, A. E. (2014). Idelalisib for the treatment of chronic lymphocytic leukemia. ISRN Oncol. 2014, 931858. doi: $10.1155 / 2014 / 931858$

Landau, D. A., Carter, S. L., Stojanov, P., McKenna, A., Stevenson, K., Lawrence, M. S., et al. (2013). Evolution and impact of subclonal mutations in chronic lymphocytic leukemia. Cell 152, 714-726. doi: 10.1016/j.cell.2013.01.019

Lespagnol, A., Duflaut, D., Beekman, C., Blanc, L., Fiucci, G., Marine, J. C., et al. (2008). Exosome secretion, including the DNA damage-induced p53-dependent secretory pathway, is severely compromised in TSAP6/Steap3-null mice. Cell Death Differ. 15, 1723-1733. doi: 10.1038/cdd.2008.104

Matta, J., Baratin, M., Chiche, L., Forel, J. M., Cognet, C., Thomas, G., et al. (2013). Induction of B7-H6, a ligand for the natural killer cell-activating receptor NKp30, in inflammatory conditions. Blood 122, 394-404. doi: 10.1182/blood2013-01-481705

Morgado, S., Sanchez-Correa, B., Casado, J. G., Duran, E., Gayoso, I., Labella, F., et al. (2011). NK cell recognition and killing of melanoma cells is controlled by multiple activating receptor-ligand interactions. J. Innate Immun. 3, 365-373. doi: $10.1159 / 000328505$

Nakayama, T., Hieshima, K., Nagakubo, D., Sato, E., Nakayama, M., Kawa, K., et al. (2004). Selective induction of Th2-attracting chemokines CCL17 and CCL22 in human B cells by latent membrane protein 1 of Epstein-Barr virus. J. Virol. 78, 1665-1674. doi: 10.1128/JVI.78.4.1665-1674.2004

Norman, J. M., Mashiba, M., McNamara, L. A., Onafuwa-Nuga, A., Chiari-Fort, E., Shen, W., et al. (2011). The antiviral factor APOBEC3G enhances the recognition of HIV-infected primary T cells by natural killer cells. Nat. Immunol. 12, 975-983. doi: 10.1038/ni.2087

Nuckel, H., Rebmann, V., Durig, J., Duhrsen, U., and Grosse-Wilde, H. (2005). HLA-G expression is associated with an unfavorable outcome and immunodeficiency in chronic lymphocytic leukemia. Blood 105, 1694-1698. doi: 10.1182/ blood-2004-08-3335

Nuckel, H., Switala, M., Sellmann, L., Horn, P. A., Durig, J., Duhrsen, U., et al. (2010). The prognostic significance of soluble NKG2D ligands in B-cell chronic lymphocytic leukemia. Leukemia 24, 1152-1159. doi: 10.1038/leu. 2010.74

Pallasch, C. P., and Hallek, M. (2014). Incorporating targeted agents into future therapy of chronic lymphocytic leukemia. Semin. Hematol. 51, 235-248. doi: 10.1053/j.seminhematol.2014.05.005

Pedersen, I. M., Kitada, S., Leoni, L. M., Zapata, J. M., Karras, J. G., Tsukada, N., et al. (2002). Protection of CLL B cells by a follicular dendritic cell line is dependent on induction of Mcl-1. Blood 100, 1795-1801.
Pogge von Strandmann, E., Simhadri, V. R., von Tresckow, B., Sasse, S., Reiners, K. S., Hansen, H. P., et al. (2007). Human leukocyte antigen-B-associated transcript 3 is released from tumor cells and engages the NKp30 receptor on natural killer cells. Immunity 27, 965-974. doi: 10.1016/j.immuni.2007.10.010

Puente, X. S., Pinyol, M., Quesada, V., Conde, L., Ordonez, G. R., Villamor, N., et al. (2011). Whole-genome sequencing identifies recurrent mutations in chronic lymphocytic leukaemia. Nature 475, 101-105. doi: 10.1038/nature10113

Quesada, V., Conde, L., Villamor, N., Ordonez, G. R., Jares, P., Bassaganyas, L., et al. (2011). Exome sequencing identifies recurrent mutations of the splicing factor SF3B1 gene in chronic lymphocytic leukemia. Nat. Genet. 44, 47-52. doi: 10.1038/ng.1032

Raulet, D. H. (2006). Missing self recognition and self tolerance of natural killer (NK) cells. Semin. Immunol. 18, 145-150. doi: 10.1016/j.smim.2006.03.003

Reiners, K. S., Topolar, D., Henke, A., Simhadri, V. R., Kessler, J., Sauer, M., et al. (2013). Soluble ligands for NK cell receptors promote evasion of chronic lymphocytic leukemia cells from NK cell anti-tumor activity. Blood 121, 36583665. doi: 10.1182/blood-2013-01-476606

Reinhardt, H. C., and Yaffe, M. B. (2009). Kinases that control the cell cycle in response to DNA damage: Chk1, Chk2, and MK2. Curr. Opin. Cell Biol. 21, 245255. doi: 10.1016/j.ceb.2009.01.018

Riches, J. C., and Gribben, J. G. (2013). Advances in chimeric antigen receptor immunotherapy for chronic lymphocytic leukemia. Discov. Med. 16, 295-302.

Robak, T. (2013). Emerging monoclonal antibodies and related agents for the treatment of chronic lymphocytic leukemia. Future Oncol. 9, 69-91. doi: 10.2217/ fon. 12.157

Rothe, A., Jachimowicz, R. D., Borchmann, S., Madlener, M., Kessler, J., Reiners, K. S., et al. (2014). The bispecific immunoligand ULBP2-aCEA redirects natural killer cells to tumor cells and reveals potent anti-tumor activity against colon carcinoma. Int. J. Cancer 134, 2829-2840. doi: 10.1002/ijc.28609

Salih, H. R., Holdenrieder, S., and Steinle, A. (2008). Soluble NKG2D ligands: prevalence, release, and functional impact. Front. Biosci. 13:3448-3456. doi: $10.2741 / 2939$

Seiffert, M., Dietrich, S., Jethwa, A., Glimm, H., Lichter, P., and Zenz, T. (2012). Exploiting biological diversity and genomic aberrations in chronic lymphocytic leukemia. Leuk. Lymphoma 53, 1023-1031. doi: 10.3109/10428194.2011. 631638

Simhadri, V. R., Reiners, K. S., Hansen, H. P., Topolar, D., Simhadri, V. L., Nohroudi, K., et al. (2008). Dendritic cells release HLA-B-associated transcript3 positive exosomes to regulate natural killer function. PLOS ONE 3:e3377. doi: 10.1371/journal.pone.0003377

Simpson, A., and Caballero, O. (2014). Monoclonal antibodies for the therapy of cancer. BMC Proc. 8(Suppl. 4), C7-O6; 1-3. doi: 10.1186/1753-6561-8-S4-O6

Singer, H., Kellner, C., Lanig, H., Aigner, M., Stockmeyer, B., Oduncu, F., et al. (2010). Effective elimination of acute myeloid leukemic cells by recombinant bispecific antibody derivatives directed against CD33 and CD16. J. Immunother. 33, 599-608. doi: 10.1097/CJI.0b013e3181dda225

Soriani, A., Zingoni, A., Cerboni, C., Iannitto, M. L., Ricciardi, M. R., Di Gialleonardo, V., et al. (2009). ATM-ATR-dependent up-regulation of DNAM-1 and NKG2D ligands on multiple myeloma cells by therapeutic agents results in enhanced NK-cell susceptibility and is associated with a senescent phenotype. Blood 113, 3503-3511. doi: 10.1182/blood-2008-08-173914

Stein, C., Kellner, C., Kugler, M., Reiff, N., Mentz, K., Schwenkert, M., et al. (2010). Novel conjugates of single-chain Fv antibody fragments specific for stem cell antigen CD123 mediate potent death of acute myeloid leukaemia cells. $\mathrm{Br}$. J. Haematol. 148, 879-889. doi: 10.1111/j.1365-2141.2009.08033.x

ten Hacken, E., and Burger, J. A. (2014). Molecular pathways: targeting the microenvironment in chronic lymphocytic leukemia--focus on the B-cell receptor. Clin. Cancer Res. 20, 548-556. doi: 10.1158/1078-0432.CCR-13-0226

Pogge von Strandmann, E. P., Hansen, H. P., Reiners, K. S., Schnell, R., Borchmann, P., Merkert, S., et al. (2006). A novel bispecific protein (ULBP2-BB4) targeting the NKG2D receptor on natural killer (NK) cells and CD138 activates NK cells and has potent antitumor activity against human multiple myeloma in vitro and in vivo. Blood 107, 1955-1962. doi: 10.1182/blood-2005-05-2177

Vyas, M., Koehl, U., Hallek, M., and Pogge von Strandmann, E. (2014). Natural ligands and antibody-based fusion proteins: harnessing the immune system against cancer. Trends Mol. Med. 20, 72-82. doi: 10.1016/j.molmed.2013. 10.006

Weston, V. J., Oldreive, C. E., Skowronska, A., Oscier, D. G., Pratt, G., Dyer, M. J., et al. (2010). The PARP inhibitor olaparib induces significant killing of 
ATM-deficient lymphoid tumor cells in vitro and in vivo. Blood 116, 4578-4587. doi: 10.1182/blood-2010-01-265769

Yair, H., Chava, P., and Ben-Zion, K. (2014). The microenvironment as a target for therapy in chronic lymphocytic leukemia. Austin. J. Cancer Clin. Res. 1, 7.

Zenz, T., Vollmer, D., Trbusek, M., Smardova, J., Benner, A., Soussi, T., et al. (2010). TP53 mutation profile in chronic lymphocytic leukemia: evidence for a disease specific profile from a comprehensive analysis of 268 mutations. Leukemia 24, 2072-2079. doi: 10.1038/leu.2010.208

Ziegler, H. W., Kay, N. E., and Zarling, J. M. (1981). Deficiency of natural killer cell activity in patients with chronic lymphocytic leukemia. Int. J. Cancer 27, 321-327. doi: 10.1002/ijc.2910270310

Conflict of Interest Statement: The authors declare that the research was conducted in the absence of any commercial or financial relationships that could be construed as a potential conflict of interest.
Received: 01 December 2014; accepted: 10 January 2015; published online: 04 February 2015.

Citation: Shatnyeva OM, Hansen HP, Reiners KS, Sauer M, Vyas M and Pogge von Strandmann E (2015) DNA damage response and evasion from immunosurveillance in CLL: new options for NK cell-based immunotherapies. Front. Genet. 6:11. doi: 10.3389/fgene.2015.00011

This article was submitted to Cancer Genetics, a section of the journal Frontiers in Genetics.

Copyright (c) 2015 Shatnyeva, Hansen, Reiners, Sauer, Vyas and Pogge von Strandmann. This is an open-access article distributed under the terms of the Creative Commons Attribution License (CC BY). The use, distribution or reproduction in other forums is permitted, provided the original author(s) or licensor are credited and that the original publication in this journal is cited, in accordance with accepted academic practice. No use, distribution or reproduction is permitted which does not comply with these terms. 